OPEN ACCESS

Edited by:

Petra Imhof,

University of Erlangen

Nuremberg, Germany

Reviewed by:

Leonid Sazanov,

Institute of Science and Technology

Austria (IST Austria), Austria

Freddy Fernandes Guimaraes,

Universidade Federal de Goiás, Brazil

Oscar Juarez,

Illinois Institute of Technology,

United States

*Correspondence:

Volker Zickermann

zickermann@med.uni-frankfurt.de

Specialty section: This article was submitted to

Theoretical and Computational

Chemistry,

a section of the journal

Frontiers in Chemistry

Received: 26 February 2021 Accepted: 06 April 2021 Published: 30 April 2021

Citation:

Galemou Yoga E, Schiller J and

Zickermann V (2021) Ubiquinone Binding and Reduction by Complex 1-Open Questions and Mechanistic Implications. Front. Chem. 9:672851.

doi: 10.3389/fchem.2021.672851

\section{Ubiquinone Binding and Reduction by Complex I-Open Questions and Mechanistic Implications}

\author{
Etienne Galemou Yoga ${ }^{1,2}$, Jonathan Schiller ${ }^{1,2}$ and Volker Zickermann ${ }^{1,2 *}$ \\ ${ }^{1}$ Institute of Biochemistry II, University Hospital, Goethe University, Frankfurt, Germany, ${ }^{2}$ Centre for Biomolecular Magnetic \\ Resonance, Institute for Biophysical Chemistry, Goethe University, Frankfurt, Germany
}

$\mathrm{NADH}$ : ubiquinone oxidoreductase (complex I) is the first enzyme complex of the respiratory chain. Complex I is a redox-driven proton pump that contributes to the proton motive force that drives ATP synthase. The structure of complex I has been analyzed by $\mathrm{x}$-ray crystallography and electron cryo-microscopy and is now well-described. The ubiquinone $(\mathrm{Q}$ ) reduction site of complex I is buried in the peripheral arm and a tunnel-like structure is thought to provide access for the hydrophobic substrate from the membrane. Several intermediate binding positions for $Q$ in the tunnel were identified in molecular simulations. Structural data showed the binding of native $Q$ molecules and short chain analogs and inhibitors in the access pathway and in the $Q$ reduction site, respectively. We here review the current knowledge on the interaction of complex I with $Q$ and discuss recent hypothetical models for the coupling mechanism.

\begin{abstract}
Keywords: respiratory chain, NADH dehydrogenase, oxidative phosphorylation, proton pumping, electron transfer, semiquinone, inhibitor
\end{abstract}

\section{INTRODUCTION}

Respiratory complex I (also known as NADH dehydrogenase or NDH-1) is a very large membrane protein found in the inner mitochondrial membrane and in the plasma membrane of aerobic bacteria (Hirst, 2013; Sazanov, 2015; Galemou Yoga et al., 2020a). Complex I couples electron transfer from NADH to quinone $(\mathrm{Q})$ to the translocation of protons across the bioenergetic membrane. Note that some bacterial species utilize menaquinone instead of ubiquinone. With a pump stoichiometry of $4 \mathrm{H}^{+}$per NADH consumed, complex I contributes substantially to the proton motive force that drives ATP synthase. A large variety of compounds are known to inhibit complex I activity by interfering with Q reduction (Murai and Miyoshi, 2016). The catalytic reaction of complex I is fully reversible. In the presence of a reduced $Q$ pool and a sufficiently high membrane potential, complex I can reduce $\mathrm{NAD}^{+}$by reverse electron transfer (RET), e.g., during reperfusion after ischemia (Chouchani et al., 2014). Mitochondrial complex I from many species can switch reversibly from an active A-form to a deactive D-form (Kotlyar and Vinogradov, 1990). The $\mathrm{A} / \mathrm{D}$ transition is thought to limit the release of detrimental oxygen species under conditions that promote RET (Drose et al., 2016). Complex I dysfunction is associated with neuromuscular and neurodegenerative diseases (Rodenburg, 2016; Fiedorczuk and Sazanov, 2018). The structure of complex I has been determined by x-ray crystallography (Baradaran et al., 2013; Zickermann et al., 2015) and by electron cryo-microscopy (cryo-EM) (Fiedorczuk et al., 2016; Zhu et al., 2016; Agip et al., 2019; Parey et al., 2019, 2020; Grba and Hirst, 2020; Kampjut and Sazanov, 2020; Soufari et al., 2020; Klusch et al., 2021). Cryo-EM structures of the related NADH dehydrogenase-like 
(ndh) complex or "photosynthetic complex I" have been reported recently (Laughlin et al., 2019; Schuller et al., 2019; Pan et al., 2020). The L-shaped architecture of complex I is highly conserved and consists of a peripheral arm (PA) and a membrane arm (MA) (Figure 1). Fourteen complex I subunits are conserved from bacteria to human. These so-called central subunits harbor all bioenergetic core functions of the enzyme complex. Eukaryotic complex I is much larger than its bacterial counterpart and comprises some 30 additional accessory subunits. The central subunits can be divided into three functional modules. The NADH oxidation module ( $\mathrm{N}$ module) and the ubiquinone reduction module ( $\mathrm{Q}$ module) constitute the $\mathrm{PA}$, whereas the proton pumping module ( $\mathrm{P}$ module) forms the $\mathrm{MA}$ of the enzyme. Eight to nine FeS clusters are found in the PA depending on the species. Seven of them connect the primary electron acceptor FMN to the $\mathrm{Q}$ reduction site, which is formed by the NDUFS2 and NDUFS7 subunits. Five FeS clusters typically give rise to electron paramagnetic resonance (EPR) signals, namely N1b, N2, N3, N4, and N5 (Ohnishi, 1998; Hirst and Roessler, 2016). Cluster N2 is the last cluster of the electron transfer chain in the PA and the immediate electron donor for Q. The membrane arm of complex I consists of seven central subunits. The three largest subunits ND2, ND4, and ND5 are related to each other and to subunits of bacterial Mrp type sodium proton antiporters (Mathiesen and Högerhäll, 2002). A hydrophilic axis (Baradaran et al., 2013) of titratable residues extending from subunit ND1 at the PA/MA interface to subunit ND5 at the distal end of the MA is thought to play a key role in energy transmission

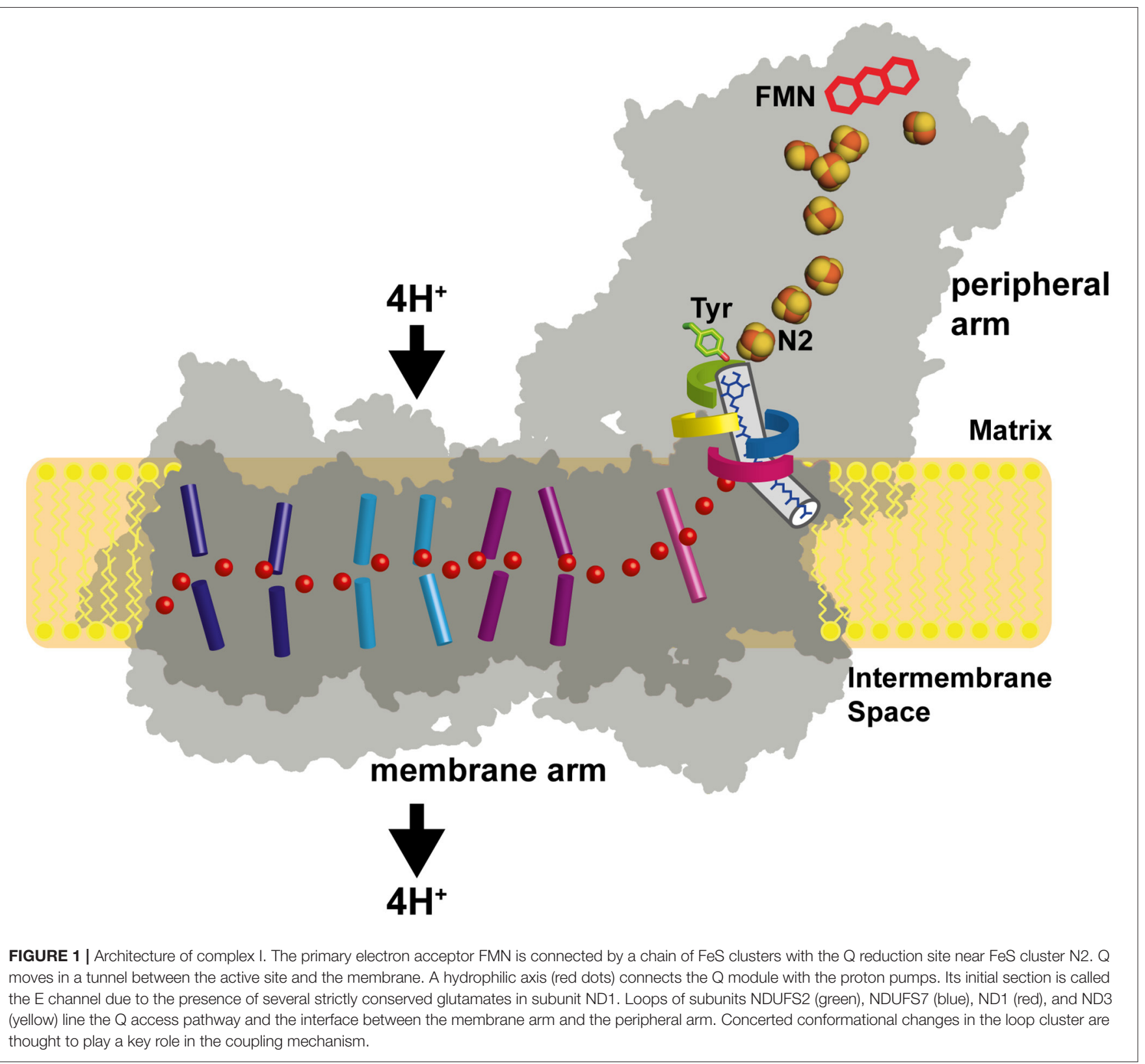


from the Q module to the pump sites. Its connection with the $\mathrm{Q}$ module is also called the $\mathrm{E}$ channel due to the presence of strictly conserved glutamate residues in ND1. We have proposed that a concerted rearrangement of loops in subunits NDUFS2, $\mathrm{ND} 1$, and ND3 is critical for converting the energy released during Q reduction into pump strokes (Zickermann et al., 2015). Indeed, there is now increasing experimental evidence for conformational changes in the Q module and at the PA/MA interface (Agip et al., 2018; Cabrera-Orefice et al., 2018; Galemou Yoga et al., 2019; Grba and Hirst, 2020; Gutierrez-Fernandez et al., 2020; Kampjut and Sazanov, 2020).

In this review, we focus on the progress in the understanding of $\mathrm{Q}$ binding and reduction by complex I and its mechanistic implications.

\section{THE Q REDUCTION SITE AND THE ACCESS PATHWAY FOR THE SUBSTRATE FROM THE MEMBRANE}

The position of the $\mathrm{Q}$ reduction site in complex $\mathrm{I}$ is unique among energy-converting Q-reactive enzymes because cluster $\mathrm{N} 2$, the immediate electron donor for $\mathrm{Q}$, resides at around 30 $\AA$ above the membrane surface (Figures 1, 2A) (Zickermann et al., 2003). Site-directed mutagenesis studies identified critical residues for Q and inhibitor binding in subunits NDUFS2 and NDUFS7 of the PA (Fendel et al., 2008; Tocilescu et al., 2010b; Sinha et al., 2015). A strictly conserved tyrosine of NDUFS2 was identified to bind the Q head group (Tocilescu et al., 2010a). The $\mathrm{x}$-ray structure of complex I from Thermus thermophilus provided structural evidence for the coordination of $\mathrm{Q}$ by this tyrosine and by a histidine residue in the loop connecting the first and the second strand of the N-terminal $\beta$-sheet of NDUFS2 (Figure 2B) (Baradaran et al., 2013; Gutierrez-Fernandez et al., 2020). This Q binding site is connected by a $\sim 35 \AA$ long tunnel with the membrane bilayer (Baradaran et al., 2013). Sitedirected mutagenesis of several residues in the Q access pathway drastically impaired Q reductase activity (Angerer et al., 2012). The entry portal of the $\mathrm{Q}$ tunnel is formed by transmembrane helices (TMHs) 1 and 6 and surface helix $\alpha 1$ of subunit ND1. Exchange of an alanine residue in helix $\alpha 1$ interferes with $Q$ reduction kinetics (Zickermann et al., 1998) and is one of the most prevalent causes for Leber's hereditary optic neuropathy (Howell et al., 1991). It has recently been suggested that the entry to the tunnel is so narrow that a conformational change is required to enable the passage of a $\mathrm{Q}$ molecule (Wang et al., 2021). The middle of the tunnel is characterized by a highly charged region formed by residues of the TMH5-6 loop of subunit ND1 and a long loop of NDUFS7. Recently, site-directed mutagenesis combined with molecular dynamics simulations identified the critical role of the NDUFS7 loop for binding and the dynamics of Q in the tunnel (Galemou Yoga et al., 2019). Fedor et al. (2017) investigated the impact of the isoprenoid chain length on the kinetics of $Q$ reduction and showed that in contrast to short-chain $\mathrm{Q}$ analogs, the dissociation of the longchain Q10 is not rate-limiting. Movement in the narrow tunnel is thought to be guided by the $\sim 50$ - $\AA$ long isoprenoid chain of
Q10 that still reaches into the membrane bilayer when the Q head group is bound at its reduction site near cluster N2. The dynamics of $\mathrm{Q}$ in the tunnel have been further studied in three computational approaches (Warnau et al., 2018; Haapanen et al., 2019; Hoias Teixeira and Menegon Arantes, 2019). Free energy profiles consistently suggested the presence of up to five different transient $\mathrm{Q}$ binding sites along the $\mathrm{Q}$ tunnel. We here follow the nomenclature for intermediate binding sites introduced by Haapanen and Sharma (denoted by Arabic numbers in Figure 2) (Haapanen et al., 2019). Interestingly, these sites largely match with $\mathrm{Q}$ binding und in complex I structures determined under different conditions (denoted by Roman numbers in Figure 2). Site 1 is close to cluster N2 at the deepest end of the Q tunnel. Hydrogen bonding of the $\mathrm{Q}$ head group with the conserved tyrosine residue (Figure 2B) was reported for crystal structures of complex I from T. thermophilus, which was soaked with the short-chain Q analog decyl benzoquinone (DBQ) (Baradaran et al., 2013; Gutierrez-Fernandez et al., 2020). A distance of around $5 \AA$ between the Q head group and tyrosine was recently observed in ovine complex I (Figure 2C) (Kampjut and Sazanov, 2020). This position is similar to the Q binding site identified in the cryo-EM structure of complex I from Yarrowia lipolytica captured under turnover (Parey et al., 2018). Although the Q head group has moved away from the tyrosine, this binding position is still assigned to site 1 . The two different binding modes might reflect the reduction of Q (Kampjut and Sazanov, 2020) and/or different functional states of the site (see below) (Parey et al., 2018). Site 3 is located approximately in the middle of the Q tunnel and shows some correlation with a bound plastoquinone (PL9) modeled in the recent cryo-EM structure of photosynthetic complex I from Thermosynechococcus elongatus (Figure 2D) (Pan et al., 2020). Site 4 is situated in the charged region in the kink of the tunnel at the PA/MA interface. A native Q9 molecule has been observed at this position in complex I from $Y$. lipolytica purified in the detergent lauryl maltose neopentylglycol (LMNG) (Figure 2E) (Parey et al., 2019). Native Q molecules were found in a similar position in plant complex I (Soufari et al., 2020; Klusch et al., 2021). In contrast, a detergent molecule was modeled at this position in complex I from $Y$. lipolytica purified in dodecyl maltoside (DDM) (Grba and Hirst, 2020). Obviously, the more bulky LMNG is unable to enter the narrow opening of the $\mathrm{Q}$ tunnel. In the closed state of ovine complex I during turnover, a second DBQ molecule was modeled close to site 4 (Figure 2F). Note that the simultaneous presence of Q molecules in site 2 and site 4 seems only possible because DBQ was used as a substrate. Steric clashes between two Q10 molecules would render a comparable scenario highly unlikely under physiological conditions (Kampjut and Sazanov, 2020). In the open state of ovine complex I with $\mathrm{NADH}$ bound, a DBQ molecule was also observed in site 4 . It is interesting to note the conformational change of the NDUFS7 loop in this structure (residue $\mathrm{R}_{2}$ in Figure 2G). A rearrangement of this loop connected with $\mathrm{Q}$ dynamics has been proposed previously (Galemou Yoga et al., 2019). Site 5 is closer to the Q tunnel entrance and binding of $\mathrm{Q}$ in this site was observed in the open conformation of ovine complex I during turnover (Figure $\mathbf{2 H}$ ) (Kampjut and Sazanov, 2020). 

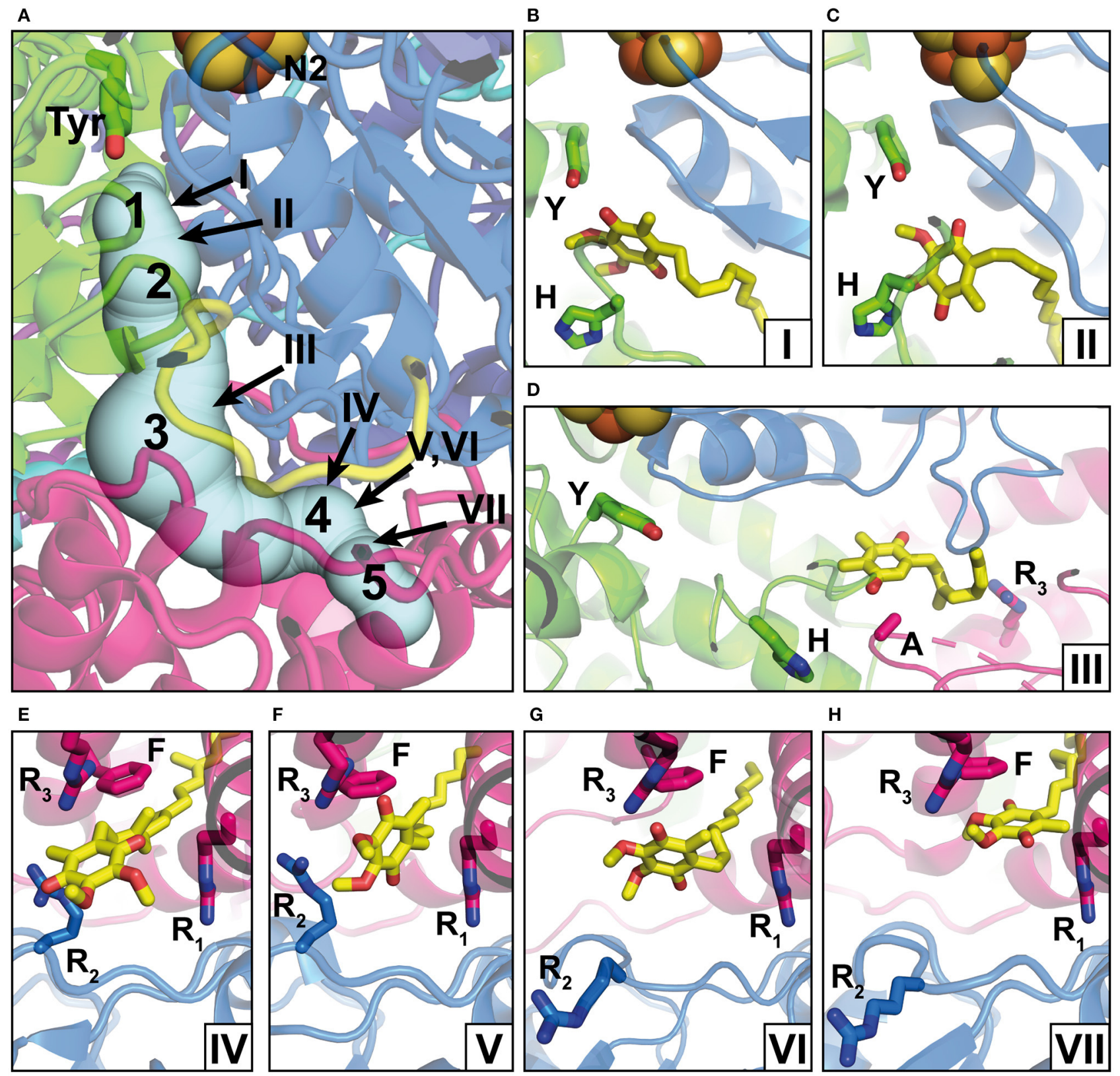

FIGURE 2 | Q binding positions. (A) The Q reduction site in the peripheral arm of complex I (PDB: 6RFR) is formed by subunits NDUFS2 (green) and NDUFS7 (blue). A tunnel for $\mathrm{Q}$ access from the membrane traverses subunit ND1 (pink). The tunnel was calculated using the CAVER3 software (Chovancova et al., 2012) (starting point conserved Y144, PDB: 6RFR, probe radius 1.3 A). Intermediate Q binding positions determined by computational methods are indicated by Arabic numbers according to Haapanen et al. (2019). The positions of Q molecules (head group) modeled into X-ray or cryo-EM structures are indicated by Roman numbers and are shown in detailed views in separate panels; the direction of view is consistent for panels in the same row. (B) DBQ bound to complex I from T. thermophilus (PDB: 6IOD) (Y, Y87; H, H38); (C) DBQ bound to complex I from Ovis aries in the closed state during turnover (PDB: 6ZKC) (Y, Y108; H, H59); (D) PL9 bound to ndh complex I from T. elongatus BP-1 (PDB: 6KHJ) (Y, Y72; H, H23; A, A237; R3, R329); (E) Q9 bound to complex I from Y. lipolytica (PDB: 6RFR) (R, R27; R2, R108, R3, R297; F, F228); (F) DBQ bound to complex I from O. aries in the closed state during turnover (PDB: 6ZKC) ( $R_{1}, R_{2} 5 ; R_{2}, R 77, R_{3}, R 274 ; F$, F224); (G) DBQ bound to complex I from O. aries in the open state with NADH bound in the $N$ module (PDB 6ZKH) $\left(R_{1}, R 25 ; R_{2}, R 77, R_{3}, R 274 ; F, F 224\right) ;(H) ~ D B Q$ bound to complex I from O. aries in the open state during turnover (PDB 6ZKD) $\left(R_{1}, R 25 ; R_{2}, R 77, R_{3}, R 274 ; F, F 224\right)$.

Taken together, the evidence for a single narrow $\mathrm{Q}$ access pathway in complex I seems to be compelling. However, it should be noted that Uno et al. (2020) showed inhibitor-sensitive reduction of $\mathrm{Q}$ analogs which are too bulky to enter the $\mathrm{Q}$ tunnel. It is currently unclear how these results can be reconciled with the structural data and more work is needed to resolve this issue. 


\section{INHIBITOR BINDING SITES IN COMPLEX I}

Complex I is known to be sensitive to a variety of inhibitors such as piericidins, rotenoids, or quinazolines (Degli Esposti, 1998; Murai and Miyoshi, 2016). Inhibitor binding to complex I was characterized by Scatchard analysis (Gutman et al., 1970), fluorescence quench titrations (Okun et al., 1999), mutagenesis (Darrouzet et al., 1998; Fendel et al., 2008; Tocilescu et al., 2010b; Sinha et al., 2015), and chemical biology approaches (Murai and Miyoshi, 2016; Uno et al., 2018). In recent years, an increasing number of complex I structures with bound inhibitors have become available (Figure 3) (Baradaran et al., 2013; Zickermann et al., 2015; Bridges et al., 2020; Gutierrez-Fernandez et al., 2020; Kampjut and Sazanov, 2020). The binding of three different inhibitors to complex I from $T$. thermophilus was recently analyzed by the Sazanov group (Gutierrez-Fernandez et al., 2020). The crystal structure of the enzyme with bound aureothin, pyridaben, and piericidin A revealed that these inhibitors bind at site 1 at the deepest end of the Q tunnel with their head groups interacting with the essential tyrosine residue near cluster N2. In the crystal structure of complex I from Y. lipolytica, the $\mathrm{Q}$ antagonist inhibitor 2-decyl-4-quinazolinyl amine (DQA) was modeled near the $\beta 1 \beta 2$ loop of the $49 \mathrm{kDa}$ subunit (Zickermann et al., 2015). More recently, the Hirst group determined the cryo-EM structure of mouse complex I with bound piericidin A (Bridges et al., 2020). Interestingly, two piericidin molecules were found in the Q tunnel. The first inhibitor molecule was bound in site 1 (Figure 3B) in agreement with the position observed in complex I from T. thermophilus, while the second molecule was observed at site 4 (not shown). This suggests that the binding of piericidin is cooperative and that piericidin competes with Q for two different binding sites in the $\mathrm{Q}$ tunnel. Since rotenone is much bulkier than other Q site inhibitors such as DQA or Piericidin, it has been hypothesized that it cannot enter and transit the narrow Q tunnel. However, in ovine complex I, two rotenone molecules were modeled in the $\mathrm{Q}$ tunnel at sites 1 (Figure 3A) and 4 (Figure 3C), respectively. Surprisingly, a third rotenone molecule was found in the ND4 subunit. Note that rotenone has been shown to inhibit $\mathrm{Na}^{+} / \mathrm{H}^{+}$antiporter activity of deactive complex I (Roberts and Hirst, 2012). The binding of rotenone in the ND4 site could explain this observation. Rotenone binding in the $\mathrm{Q}$ tunnel suggests either that the entrance of the $\mathrm{Q}$ tunnel undergoes a reorganization to allow access of the bulky molecule or that rotenone can access the Q site via alternative pathways (Uno et al., 2018).

\section{Q REDUCTION AND MECHANISTIC IMPLICATIONS}

It is generally accepted that the energy for driving the proton pumps is released in the $\mathrm{Q}$ module and there is increasing evidence showing that the concerted rearrangement of a cluster of four loops surrounding the $\mathrm{Q}$ binding site and at the interface of PA and MA (Figure 1) is a central element of the coupling mechanism (Zickermann et al., 2015; Cabrera-Orefice et al., 2018; Parey et al., 2018; Galemou Yoga et al., 2019, 2020b; Kampjut and Sazanov, 2020). Full reduction of Q requires the uptake of two electrons and two protons. The delivery of electrons by a single electron donor and the observation of semiquinone radicals by EPR spectroscopy (Magnitsky et al., 2002) fostered the idea that reaction intermediates accumulate in a stepwise reaction sequence from $\mathrm{Q}$ to $\mathrm{QH}_{2}$. Early on, the formation of negatively charged quinone species has been proposed to be a key step in the catalytic cycle (Euro et al., 2008). However, note that more recently the assignment of semiquinone EPR signals to complex I has been questioned (Wright et al., 2020). In any case, the timing
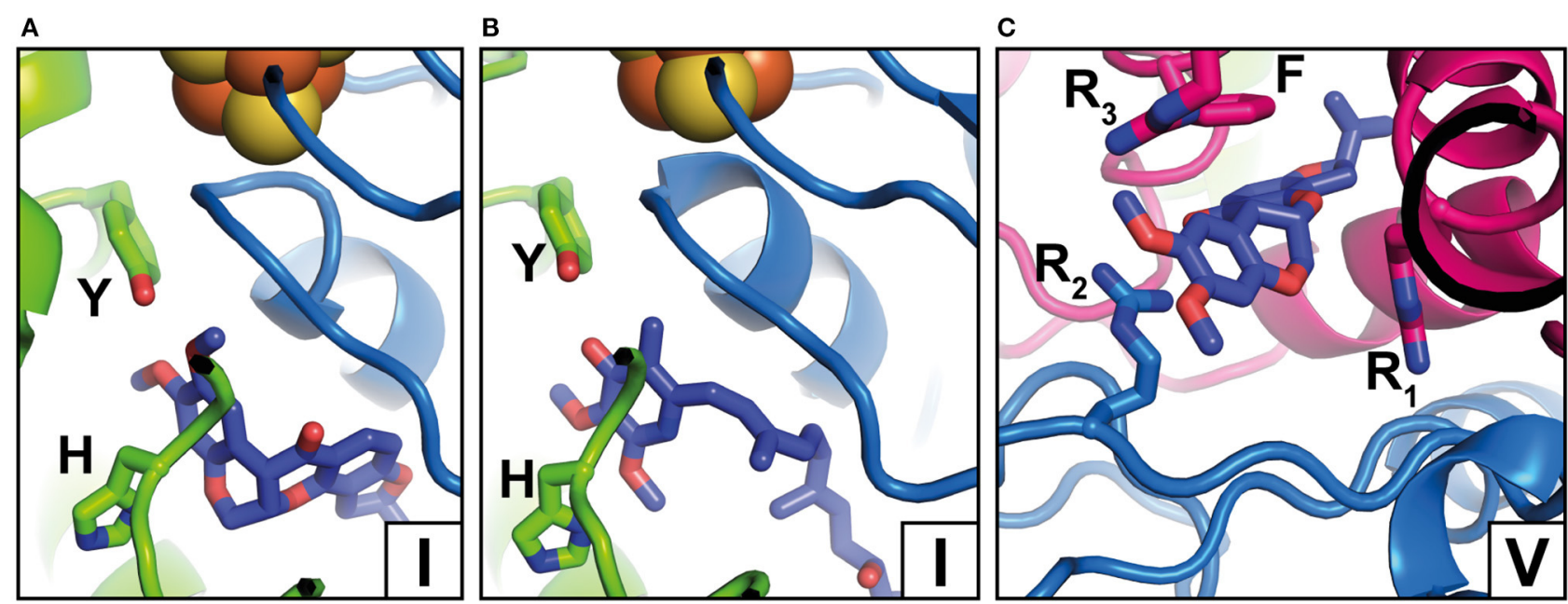

FIGURE 3 | Inhibitor binding positions. (A) Rotenone bound to complex I from O. aries in the closed state (PDB 6ZKK) (Y, Y108; H, H59); (B) Piericidin A bound to complex I from Mus musculus (PDB 6ZTQ) (Y, Y108; H, H59); (C) Rotenone bound to complex I from O. aries in the open state (PDB 6ZKL) (R1, R25; $R_{2}, R 77, R_{3}$, R274; F, F224). Binding positions in the $Q$ tunnel are indicated by Roman numbers and correspond to Figure $\mathbf{2} \mathbf{A}$ but note that rotenone occupies a larger area. For colors, see the legend of Figure 2. 
of charge movements and charge compensation reactions in the $\mathrm{Q}$ reduction site is thought to be of utmost importance for the coupling mechanism. Over the years, a number of mechanistic schemes for redox-linked proton translocation have been proposed and a selection of recent models is discussed below.

The stabilization of negatively charged intermediates of $\mathrm{Q}$ redox chemistry $\left(\mathrm{Q}^{-}\right.$and $\left.\mathrm{QH}^{-}\right)$plays a central role in the two-state stabilization-change mechanism proposed by Brandt (Brandt, 2011). This mechanism assumes two different functional states of the Q binding site. Electron transfer from cluster N2 to $\mathrm{Q}$ or $\mathrm{QH}$ is only possible in the so-called $\mathrm{E}$ state. Proton transfer to $\mathrm{Q}^{-}$or $\mathrm{QH}^{-}$is only possible in the so-called $\mathrm{P}$ state. Stabilization of the anionic species generated in the E state provides the energy for proton pumping and is tightly linked with transition from the $\mathrm{E}$ to the $\mathrm{P}$ state. Full reduction of $\mathrm{Q}$ to $\mathrm{QH}_{2}$ includes two E-to-P state transformations. This does not necessarily mean the execution of two separate pump events because transient storage of electrostatic or conformational energy would still allow pumping in a single step. The cycling between the $\mathrm{E}$ and $\mathrm{P}$ states is thought to involve a conformational rearrangement of the $\mathrm{Q}$ reduction site (Zickermann et al., 2015; Cabrera-Orefice et al., 2018). In fact, we have observed a different mode of $\mathrm{Q}$ binding in the cryo-EM structure of complex I from Y. lipolytica captured during steady-state activity as compared with Q binding observed in native complex I from T. thermophilus (Parey et al., 2018). Different conformations of the $\beta 1 \beta 2$ loop of NDUFS2 in both structures further support the idea of a two-state mechanism associated with concerted loop rearrangements. Recent structures of ovine (Kampjut and Sazanov, 2020) and T. thermophilus (Gutierrez-Fernandez et al., 2020) confirm a different mode of $\mathrm{Q}$ binding to site 1 (Figures 2B,C). Nevertheless, more work is needed to establish an unequivocal link between the hypothetical $\mathrm{E}$ and $\mathrm{P}$ states with the protein structure.

Based on structural information with increasing resolution, molecular modeling and molecular dynamics simulation approaches have been established as powerful tools to study complex I function (Hummer and Wikström, 2016). In initial quantum mechanics/molecular mechanics (QM/MM) simulations of Q reduction, Sharma et al. (2015) placed a Q molecule into the $\mathrm{Q}$ reduction site and tested the impact of different $\mathrm{Q}$ redox states. In case simulations were performed with a $\mathrm{Q}^{2-}$, i.e., assuming a two-electron reduction, fast proton transfer from the coordinating tyrosine and histidine residues was observed resulting in the formation of $\mathrm{QH}_{2}$. Prior to the proton transfer reaction, the histidine residue forms a salt bridge with a conserved aspartate residue of NDUFS2 that is located further toward subunit ND1. Breaking this ion pair by the redox-coupled proton transfer to $Q$ triggers a conformational change of the $\beta 1 \beta 2$ loop and subsequent flipping of the aspartate side chain is associated with rearrangements of conserved acidic residues in the ND1 subunit. Electrostatic pKa calculations suggested that these changes result in proton uptake from the $\mathrm{N}$ side and are thus thought to trigger the loading of the proton pump. Note that the initial formation of $\mathrm{Q}^{2-}$ is an essential prerequisite for this mechanism because no proton transfer reactions were observed when oxidized Q or semiquinone states were modeled in the site.

In a later study, Gamiz-Hernandez et al. (2017) reported that the negative charge of cluster N2 shifts the midpoint potential of ubiquinone to a value in the range of $-300 \mathrm{mV}$. This value is unusually low but is in agreement with the values reported earlier based on freeze-quench reduction kinetics (Verkhovskaya et al., 2008) and electrometric calculations (Verkhovskaya and Wikström, 2014). Remarkably, such a dramatic shift in potential would result in an annihilation of the redox potential difference between $\mathrm{NADH}$ and a $\mathrm{Q}$ molecule in site 1 and would consequently render $\mathrm{Q}$ reduction isoenergetic (Wikström et al., 2015; Gamiz-Hernandez et al., 2017; Kaila, 2018). Since, in this scenario, there is a redox potential difference between $\mathrm{Q}$ in site 1 and $\mathrm{Q}$ in the membrane $(+90 \mathrm{mV})$, the release in free energy is thought to be associated with the movement of $\mathrm{Q}$ between site 1 and the exit of the Q tunnel (Wikström et al., 2015; Kaila, 2018). The binding of $\mathrm{QH}_{2}$ close to the entry of the $\mathrm{E}$ channel, corresponding approximately to site 4, is suggested to "push" out protons previously loaded on acidic ND1 residues by the mechanism described above (Kaila, 2018; Mühlbauer et al., 2020). However, the molecular details of that energy conversion step remain obscure.

The association of complex I with a tightly bound Q molecule was first reported by Verkhovsky et al. (2012) for the enzyme from Escherichia coli. Later, native Q molecules were observed in the cryo-EM structures of complex I from Y. lipolytica (Parey et al., 2019) and Brassica oleracea (Soufari et al., 2020) and free energy calculations suggested that a large energy barrier restricts the movement of $\mathrm{Q}$ from the tunnel into the membrane bilayer (Warnau et al., 2018; Haapanen et al., 2019; Hoias Teixeira and Menegon Arantes, 2019). Wikström et al. (2015) have discussed the function of a Q molecule trapped in the Q tunnel to shuttle electrons between cluster N2 and a substrate Q molecule of the membrane Q pool. Haapanen et al. (2019) proposed that the two-electron reduction of a "shuttling Q" by FeS cluster N2 and proton transfer from the nearby tyrosine leads to the formation of a $\mathrm{QH}^{-}$molecule and tyrosinate in site 1 . The repulsion of negative charges is thought to drive the movement of $\mathrm{QH}^{-}$to site 4. A substrate $\mathrm{Q}$ molecule in site 5 is reduced by electron transfer from the shuttling $\mathrm{Q}$, while the proton released in site 4 is suggested to enter the $\mathrm{E}$ channel and to push out protons loaded on antiporter-like subunits. A shuttling $Q$ is known to operate in photosystem II and in bacterial photoreaction centers (Müh et al., 2012). However, for complex I, unambiguous experimental evidence for a comparable mechanistic concept, e.g., the observation of a spin-spin coupled state between two SQ species by EPR spectroscopy, is still lacking.

The recent high-resolution structure of the ovine complex I reported by Kampjut and Sazanov (Kampjut and Sazanov, 2020) has offered a detailed view on Q binding and conformational changes in loops in the Q module and in ND1. Q reduction is thought to involve proton transfer from the $\mathrm{Q}$ coordinating histidine and tyrosine residues but in contrast to any previously proposed models, the authors of this study hypothesize that for the re-protonation of site 1, the protons are extracted 
from two acidic residues in membrane-intrinsic subunit ND4L. The resulting negative charge in this subunit is suggested to subsequently trigger a series of events in the hydrophilic axis that ultimately lead to proton translocation to the P side. However, proton transfer from the membrane interior to site 1 is at variance with two recent studies, which identified putative proton access pathways from the N side (Galemou Yoga et al., 2020b; Grba and Hirst, 2020).

Taken together, the recent surge in high-resolution structural information in combination with molecular simulations and functional studies has greatly advanced the general understanding of complex I. However, conceptually different mechanisms for redox-linked proton translocation are currently discussed. Cryo-EM techniques including tomography as well as computational approaches will become even more powerful in the future. The identification of further intermediates is an

\section{REFERENCES}

Agip, A. A., Blaza, J. N., Bridges, H. R., Viscomi, C., Rawson, S., Muench, S. P., et al. (2018). Cryo-EM structures of complex I from mouse heart mitochondria in two biochemically defined states. Nat. Struct. Mol. Biol. 25, 547-556. doi: 10.1038/s41594-018-0073-1

Agip, A. A., Blaza, J. N., Fedor, J. G., and Hirst, J. (2019). Mammalian respiratory complex I through the lens of cryo-EM. Annu. Rev. Biophys. 48, 165-184. doi: 10.1146/annurev-biophys-052118-115704

Angerer, H., Nasiri, H. R., Niedergesass, V., Kerscher, S., Schwalbe, H., and Brandt, U. (2012). Tracing the tail of ubiquinone in mitochondrial complex I. Biochim. Biophys. Acta 1817, 1776-1784. doi: 10.1016/j.bbabio.2012.03.021

Baradaran, R., Berrisford, J. M., Minhas, G. S., and Sazanov, L. A. (2013). Crystal structure of the entire respiratory complex I. Nature 494, 443-448. doi: $10.1038 /$ nature11871

Brandt, U. (2011). A two-state stabilization-change mechanism for proton-pumping complex I. Biochim. Biophys. Acta 1807, 1364-1369. doi: 10.1016/j.bbabio.2011.04.006

Bridges, H. R., Fedor, J. G., Blaza, J. N., Di Luca, A., Jussupow, A., Jarman, O. D., et al. (2020). Structure of inhibitor-bound mammalian complex I. Nat. Commun. 11:5261. doi: 10.1038/s41467-020-18950-3

Cabrera-Orefice, A., Yoga, E. G., Wirth, C., Siegmund, K., Zwicker, K., GuerreroCastillo, S., et al. (2018). Locking loop movement in the ubiquinone pocket of complex I disengages the proton pumps. Nat. Commun. 9:4500. doi: 10.1038/s41467-018-06955-y

Chouchani, E. T., Pell, V. R., Gaude, E., Aksentijevic, D., Sundier, S. Y., Robb, E. L., et al. (2014). Ischaemic accumulation of succinate controls reperfusion injury through mitochondrial ROS. Nature 515, 431-435. doi: 10.1038/nature13909

Chovancova, E., Pavelka, A., Benes, P., Strnad, O., Brezovsky, J., Kozlikova, B., et al. (2012). CAVER 3.0: a tool for the analysis of transport pathways in dynamic protein structures. PLoS. Comput. Biol. 8:e1002708. doi: 10.1371/journal.pcbi.1002708

Darrouzet, E., Issartel, J. P., Lunardi, J., and Dupuis, A. (1998). The 49-kDa subunit of NADH-ubiquinone oxidoreductase (Complex I) is involved in the binding of piericidin and rotenone, two quinone-related inhibitors. FEBS Lett. 431, 34-38. doi: 10.1016/S0014-5793(98)00719-4

Degli Esposti, M. (1998). Inhibitors of NADH-ubiquinone reductase: an overview. Biochim. Biophys. Acta 1364, 222-235. doi: 10.1016/S0005-2728(98)00029-2

Drose, S., Stepanova, A., and Galkin, A. (2016). Ischemic A/D transition of mitochondrial complex I and its role in ROS generation. Biochim. Biophys. Acta 1857, 946-957. doi: 10.1016/j.bbabio.2015.12.013

Euro, L., Belevich, G., Verkhovsky, M. I., Wikström, M., and Verkhovskaya, M. (2008). Conserved lysine residues of the membrane subunit NuoM are involved in energy conversion by the proton-pumping NADH:ubiquinone oxidoreductase (Complex I). Biochim. Biophys. Acta 1777, 1166-1172. doi: 10.1016/j.bbabio.2008.06.001 avenue for research to comprehensively understand the catalytic cycle of respiratory complex I.

\section{AUTHOR CONTRIBUTIONS}

EG, JS, and VZ wrote the paper. All authors contributed to the article and approved the submitted version.

\section{FUNDING}

This work was supported by the Deutsche Forschungsgemeinschaft (DFG grant ZI552/4-2 to VZ).

\section{ACKNOWLEDGMENTS}

We thank Vivek Sharma for the discussions.

Fedor, J. G., Jones, A. J. Y., Di, L. A., Kaila, V. R. I., and Hirst, J. (2017) Correlating kinetic and structural data on ubiquinone binding and reduction by respiratory complex I. Proc. Natl. Acad. Sci. U.S.A. 114, 12737-12742. doi: $10.1073 /$ pnas. 1714074114

Fendel, U., Tocilescu, M. A., Kerscher, S., and Brandt, U. (2008). Exploring the inhibitor binding pocket of respiratory complex I. Biochim. Biophys. Acta 1777, 660-665. doi: 10.1016/j.bbabio.2008. 04.033

Fiedorczuk, K., Letts, J. A., Degliesposti, G., Kaszuba, K., Skehel, M., and Sazanov, L. A. (2016). Atomic structure of the entire mammalian mitochondrial complex I. Nature 538, 406-410. doi: 10.1038/nature 19794

Fiedorczuk, K., and Sazanov, L. A. (2018). Mammalian mitochondrial complex I structure and disease-causing mutations. Trends Cell Biol. 28, 835-867. doi: 10.1016/j.tcb.2018.06.006

Galemou Yoga, E., Angerer, H., Parey, K., and Zickermann, V. (2020a). Respiratory complex I - Mechanistic insights and advances in structure determination. Biochim. Biophys. Acta 1861:148153. doi: 10.1016/j.bbabio.2020.148153

Galemou Yoga, E., Haapanen, O., Wittig, I., Siegmund, K., Sharma, V., and Zickermann, V. (2019). Mutations in a conserved loop in the PSST subunit of respiratory complex I affect ubiquinone binding and dynamics. Biochim. Biophys. Acta 1860, 573-581. doi: 10.1016/j.bbabio.2019.06.006

Galemou Yoga, E., Parey, K., Djurabekova, A., Haapanen, O., Siegmund, K. Zwicker, K., et al. (2020b). Essential role of accessory subunit LYRM6 in the mechanism of mitochondrial complex I. Nat. Commun. 11:6008 doi: 10.1038/s41467-020-19778-7

Gamiz-Hernandez, A. P., Jussupow, A., Johansson, M. P., and Kaila, V. R. I. (2017). Terminal electron-proton transfer dynamics in the quinone reduction of respiratory complex I. J. Am. Chem. Soc. 139, 16282-16288. doi: 10.1021 /jacs.7b08486

Grba, D. N., and Hirst, J. (2020). Mitochondrial complex I structure reveals ordered water molecules for catalysis and proton translocation. Nat. Struct. Mol. Biol. 27, 892-900. doi: 10.1038/s41594-020-0473-x

Gutierrez-Fernandez, J., Kaszuba, K., Minhas, G. S., Baradaran, R., Tambalo, M., Gallagher, D. T., et al. (2020). Key role of quinone in the mechanism of respiratory complex I. Nat. Commun. 11:4135. doi: 10.1038/s41467-020-17957-0

Gutman, M., Singer, T. P., and Casida, J. E. (1970). Studies on the respiratory chain-linked reduced nicotinamide adenine dinucleotide dehydrogenase. XVII. Reaction sites of piericidin A and rotenone. J. Biol. Chem. 245, 1992-1997. doi: 10.1016/S0021-9258(18)63196-5

Haapanen, O., Djurabekova, A., and Sharma, V. (2019). Role of second quinone binding site in proton pumping by respiratory complex I. Front. Chem. 7:221. doi: $10.3389 /$ fchem.2019.00221

Hirst, J. (2013). Mitochondrial complex I. Annu. Rev. Biochem. 82, 551-575. doi: 10.1146/annurev-biochem-070511-103700 
Hirst, J., and Roessler, M. M. (2016). Energy conversion, redox catalysis and generation of reactive oxygen species by respiratory complex I. Biochim. Biophys. Acta 1857, 872-883. doi: 10.1016/j.bbabio.2015.12.009

Hoias Teixeira, M., and Menegon Arantes, G. (2019). Balanced internal hydration discriminates substrate binding to respiratory complex I. Biochim. Biophys. Acta 1860, 541-548. doi: 10.1016/j.bbabio.2019.05.004

Howell, N., Bindoff, L. A., McCullough, D. A., Kubacka, I., Poulton, J., Mackey, D., et al. (1991). Leber hereditary optic neuropathy: identification of the same mitochondrial ND1 mutation in six pedigrees. Am. J. Hum. Genet. 49, 939-950.

Hummer, G., and Wikström, M. (2016). Molecular simulation and modeling of complex I. Biochim. Biophys. Acta 1857, 915-921. doi: 10.1016/j.bbabio.2016.01.005

Kaila, V. R. I. (2018). Long-range proton-coupled electron transfer in biological energy conversion: towards mechanistic understanding of respiratory complex I. J. R. Soc. Interface 15:20170916. doi: 10.1098/rsif.2017.0916

Kampjut, D., and Sazanov, L. A. (2020). The coupling mechanism of mammalian respiratory complex I. Science 370:eabc4209. doi: 10.1126/science.abc4209

Klusch, N., Senkler, J., Yildiz, Ö., Kühlbrandt, W., and Braun, H. P. (2021). A ferredoxin bridge connects the two arms of plant mitochondrial complex I. Plant Cell. doi: 10.1093/plcell/koab092

Kotlyar, A. B., and Vinogradov, A. D. (1990). Slow active/inactive transition of the mitochondrial NADH-ubiquinone reductase. Biochim. Biophys. Acta 1019, 151-158. doi: 10.1016/0005-2728(90)90137-S

Laughlin, T. G., Bayne, A. N., Trempe, J. F., Savage, D. F., and Davies, K. M. (2019). Structure of the complex I-like molecule NDH of oxygenic photosynthesis. Nature 566, 411-414. doi: 10.1038/s41586-019-0921-0

Magnitsky, S., Toulokhonova, L., Yano, T., Sled, V. D., Högerhäll, C., Grivennikova, V. G., et al. (2002). EPR characterization of ubisemiquinones and iron-sulfur cluster $\mathrm{N} 2$, central components of the energy coupling in the NADH-ubiquinone oxidoreductase (complex I) in situ. J. Bioenerg. Biomembr. 34, 193-208. doi: 10.1023/A:1016083419979

Mathiesen, C., and Högerhäll, C. (2002). Transmembrane topology of the NuoL, $\mathrm{M}$ and $\mathrm{N}$ subunits of NADH:quinone oxidoreductase and their homologues among membrane-bound hydrogenases and bona fide antiporters. Biochim. Biophys. Acta 1556, 121-132. doi: 10.1016/S0005-2728(02)00343-2

Müh, F., Glockner, C., Hellmich, J., and Zouni, A. (2012). Light-induced quinone reduction in photosystem II. Biochim Biophys Acta 1817, 44-65. doi: 10.1016/j.bbabio.2011.05.021

Mühlbauer, M. E., Saura, P., Nuber, F., Di Luca, A., Friedrich, T., and Kaila, V. R. I. (2020). Water-Gated Proton Transfer Dynamics in Respiratory Complex I. J. Am. Chem. Soc. 142, 13718-13728. doi: 10.1021/jacs.0c02789

Murai, M., and Miyoshi, H. (2016). Current topics on inhibitors of respiratory complex I. Biochim. Biophys. Acta 1857, 884-891. doi: 10.1016/j.bbabio.2015.11.009

Ohnishi, T. (1998). Iron-sulfur clusters/semiquinones in complex I. Biochim. Biophys. Acta 1364, 186-206. doi: 10.1016/S0005-2728(98)00027-9

Okun, J. G., Lummen, P., and Brandt, U. (1999). Three classes of inhibitors share a common binding domain in mitochondrial complex I (NADH:ubiquinone oxidoreductase). J. Biol. Chem. 274, 2625-2630. doi: 10.1074/jbc.274.5.2625

Pan, X., Cao, D., Xie, F., Xu, F., Su, X., Mi, H., et al. (2020). Structural basis for electron transport mechanism of complex I-like photosynthetic NAD(P)H dehydrogenase. Nat. Commun. 11:610. doi: 10.1038/s41467-020-1 4456-0

Parey, K., Brandt, U., Xie, H., Mills, D. J., Siegmund, K., Vonck, J., et al. (2018). Cryo-EM structure of respiratory complex I at work. Elife 7:45. doi: 10.7554/eLife.39213.045

Parey, K., Haapanen, O., Sharma, V., Kofeler, H., Zullig, T., Prinz, S., et al. (2019). High-resolution cryo-EM structures of respiratory complex I: mechanism, assembly, and disease. Sci. Adv. 5:eaax9484. doi: 10.1126/sciadv.aax9484

Parey, K., Wirth, C., Vonck, J., and Zickermann, V. (2020). Respiratory complex I - structure, mechanism and evolution. Curr. Opin. Struct. Biol. 63, 1-9. doi: 10.1016/j.sbi.2020.01.004

Roberts, P. G., and Hirst, J. (2012). The deactive form of respiratory complex I from mammalian mitochondria is a $\mathrm{Na}^{+} / \mathrm{H}^{+}$antiporter. J. Biol. Chem 287, 34743-34751. doi: 10.1074/jbc.M112.384560

Rodenburg, R. J. (2016). Mitochondrial complex I-linked disease. Biochim. Biophys. Acta 1857, 938-945. doi: 10.1016/j.bbabio.2016.02.012
Sazanov, L. A. (2015). A giant molecular proton pump: structure and mechanism of respiratory complex I. Nat. Rev. Mol. Cell. Biol. 16, 375-388. doi: 10.1038/nrm3997

Schuller, J. M., Birrell, J. A., Tanaka, H., Konuma, T., Wulfhorst, H., Cox, N., et al. (2019). Structural adaptations of photosynthetic complex I enable ferredoxin-dependent electron transfer. Science 363, 257-260. doi: $10.1126 /$ science.aau3613

Sharma, V., Belevich, G., Gamiz-Hernandez, A. P., Rog, T., Vattulainen, I., Verkhovskaya, M. L., et al. (2015). Redox-induced activation of the proton pump in the respiratory complex I. Proc. Natl. Acad. Sci. U.S.A. 112, 11571-11576. doi: 10.1073/pnas.1503761112

Sinha, P. K., Castro-Guerrero, N., Patki, G., Sato, M., Torres-Bacete, J., Sinha, S., et al. (2015). Conserved amino acid residues of the NuoD segment important for structure and function of Escherichia coli NDH-1 (complex I). Biochemistry 54, 753-764. doi: 10.1021/bi501403t

Soufari, H., Parrot, C., Kuhn, L., Waltz, F., and Hashem, Y. (2020). Specific features and assembly of the plant mitochondrial complex I revealed by cryo-EM. Nat. Commun. 11:5195. doi: 10.1038/s41467-020-18814-w

Tocilescu, M. A., Fendel, U., Zwicker, K., Drose, S., Kerscher, S., and Brandt, U. (2010a). The role of a conserved tyrosine in the $49-\mathrm{kDa}$ subunit of complex I for ubiquinone binding and reduction. Biochim. Biophys. Acta 1797, 625-632. doi: 10.1016/j.bbabio.2010.01.029

Tocilescu, M. A., Zickermann, V., Zwicker, K., and Brandt, U. (2010b). Quinone binding and reduction by respiratory complex I. Biochim. Biophys. Acta 1797, 1883-1890. doi: 10.1016/j.bbabio.2010.05.009

Uno, S., Kimura, H., Murai, M., and Miyoshi, H. (2018). Exploring the quinone/inhibitor-binding pocket in mitochondrial respiratory complex I by chemical biology approaches. J. Biol. Chem. 294, 679-696. doi: $10.1074 /$ jbc.RA118.006056

Uno, S., Masuya, T., Shinzawa-Itoh, K., Lasham, J., Haapanen, O., Shiba, T., et al. (2020). Oversized ubiquinones as molecular probes for structural dynamics of the ubiquinone reaction site in mitochondrial respiratory complex I. J. Biol. Chem. 295, 2449-2463. doi: 10.1074/jbc.RA119.012347

Verkhovskaya, M., and Wikström, M. (2014). Oxidoreduction properties of bound ubiquinone in complex I from Escherichia coli. Biochim. Biophys. Acta 1837, 246-250. doi: 10.1016/j.bbabio.2013.11.001

Verkhovskaya, M. L., Belevich, N., Euro, L., Wikstrom, M., and Verkhovsky, M. I. (2008). Real-time electron transfer in respiratory complex I. Proc.Natl.Acad.Sci. U.S.A. 105, 3763-3767. doi: 10.1073/pnas.0711249105

Verkhovsky, M., Bloch, D. A., and Verkhovskaya, M. (2012). Tightly-bound ubiquinone in the Escherichia coli respiratory complex I. Biochim. Biophys. Acta 1817, 1550-1556. doi: 10.1016/j.bbabio.2012.04.013

Wang, P., Dhananjayan, N., Hagras, M. A., and Stuchebrukhov, A. A. (2021). Respiratory complex I: bottleneck at the entrance of quinone site requires conformational change for its opening. Biochim. Biophys. Acta 1862:148326. doi: 10.1016/j.bbabio.2020.148326

Warnau, J., Sharma, V., Gamiz-Hernandez, A. P., Di Luca, A., Haapanen, O., Vattulainen, I., et al. (2018). Redox-coupled quinone dynamics in the respiratory complex I. Proc. Natl. Acad. Sci. U.S.A. 115, E8413-E8420. doi: 10.1073/pnas.1805468115

Wikström, M., Sharma, V., Kaila, V. R., Hosler, J. P., and Hummer, G. (2015). New perspectives on proton pumping in cellular respiration. Chem. Rev. 115, 2196-2221. doi: $10.1021 / \mathrm{cr} 500448 \mathrm{t}$

Wright, J. J., Fedor, J. G., Hirst, J., and Roessler, M. M. (2020). Using a chimeric respiratory chain and EPR spectroscopy to determine the origin of semiquinone species previously assigned to mitochondrial complex I. BMC Biol. 18:54. doi: 10.1186/s12915-020-00768-6

Zhu, J., Vinothkumar, K. R., and Hirst, J. (2016). Structure of mammalian respiratory complex I. Nature 536, 354-358. doi: 10.1038/nature19095

Zickermann, V., Barquera, B., Wikström, M., and Finel, M. (1998). Analysis of the pathogenic human mitochondrial mutation ND1/3460, and mutations of strictly conserved residues in its vicinity, using the bacterium Paracoccus denitrificans. Biochemistry 37, 11792-11796. doi: 10.1021/bi9810555

Zickermann, V., Bostina, M., Hunte, C., Ruiz, T., Radermacher, M., and Brandt, U. (2003). Functional implications from an unexpected position of the $49-\mathrm{kDa}$ subunit of NADH:ubiquinone oxidoreductase. J. Biol. Chem. 278, 29072-29078. doi: 10.1074/jbc.M302713200 
Zickermann, V., Wirth, C., Nasiri, H., Siegmund, K., Schwalbe, H., Hunte, C., et al. (2015). Mechanistic insight from the crystal structure of mitochondrial complex I. Science 347, 44-49. doi: 10.1126/science.1259859

Conflict of Interest: The authors declare that the research was conducted in the absence of any commercial or financial relationships that could be construed as a potential conflict of interest.
Copyright $\odot 2021$ Galemou Yoga, Schiller and Zickermann. This is an open-access article distributed under the terms of the Creative Commons Attribution License (CC $B Y)$. The use, distribution or reproduction in other forums is permitted, provided the original author(s) and the copyright owner(s) are credited and that the original publication in this journal is cited, in accordance with accepted academic practice. No use, distribution or reproduction is permitted which does not comply with these terms. 